\title{
Apoptosis and pericyte loss in alveolar capillaries in COVID-19 infection: choice of markers matters
}

\author{
Amit Jain *iD and D. John Doyle
}

(C) 2020 Springer-Verlag GmbH Germany, part of Springer Nature

\section{Dear Editor,}

We enjoyed the article by Cardot-Leccia et al. demonstrating apoptosis and pericyte loss in alveolar capillaries in COVID-19 lung [1]. Although pericytes have a significant role in COVID-19 pathobiology, we have concerns related to the experimental model used to produce this conclusion.

First, the scarcity of pericytes in the lungs and the lack of exclusive markers for reproducible histologic identification may be problematic. While $\alpha$-SMA has been described as a pericyte marker, in general, only mural cells associated with precapillary arterioles and postcapillary venules express this contractile protein, whereas quiescent capillary pericytes do not [2]. Thus, $\alpha$-SMA immunostaining should not be used to differentiate lung pericytes, and its appearance in the alveolar capillaries should not be considered as a normal finding. Increased expression of $\alpha$-SMA positive pericytes in the alveolar capillaries suggests mesenchymal-like abnormal phenotypic differentiation of pericytes as observed in pulmonary fibrosis and pulmonary hypertension [2]. Thus, the authors' finding of reduced $\alpha$-SMA immunostaining in alveolar capillaries in COVID + lung, with abundant $\alpha$-SMA pericytes in venular walls may not be far from the normal and does not necessarily signify pericyte loss in alveolar capillaries.

Second, Cardot-Leccia et al. used "normal" lung tissue from a treated pneumothorax patient as a control. However, lung inflammation and oxidative stress may be associated with spontaneous pneumothorax pathogenesis,

${ }^{*}$ Correspondence: amitvasujain@gmail.com

Anesthesiology Institute, Cleveland Clinic Abu Dhabi, Al Maryah Island, Abu Dhabi, United Arab Emirates while pleural lavage fluid in such patients has high concentrations of IL-5, IL-6, IL-8, IL-12, and TNF- $\alpha$ [3]. Surprisingly, high levels of lipopolysaccharide binding protein have also been identified. As a result, phenotypic alteration of $\alpha$-SMA-negative pericytes to mural-like $\alpha$-SMA-positive cells in the lung tissue from a patient with pneumothorax cannot be ruled out. Thus, the levels of $\alpha$-SMA immunostaining in the lung tissue from the control patient in the study [1] may be fallaciously higher than the COVID-19 patient. Also, the $\alpha$-SMA-positive cells may reflect not only pericytes but also parenchymal and mesothelial-derived myofibroblasts, which have previously been implicated in lung repair and remodeling [2].

Third, though pericytes have been identified as the cells with high expression of ACE2 in organs such as heart and brain, lung pericytes seem to be deficient in ACE2 [4]. As a result, although direct infection of pericytes (undoubtedly following endothelialitis) by SARS-CoV-2 can be a possibility in heart and brain, SARS-CoV-2 directly infecting lung pericytes is less likely. We believe that pericyte apoptosis in the inter-alveolar septum of COVID-19 patients, as revealed by cleaved caspase three [1], is secondary to ACE-Ang II-AT1R overactivity in COVID19 , resulting in increased Tie 2 activity and reduced Tie1 expression. This supports the epithelial-endothelial cross-talk hypothesis [5] of indirect pericyte and endothelial cell-injury following the downregulation of ACE2 and upregulation of IL6, TNF- $\alpha$ and AT1R expression secondary to SARS-CoV-2 infecting the pulmonary alveolar capillary epithelium. Notably, high local Ang II levels have been implicated in the pathophysiology of indirect pericyte loss and acellular capillaries in diabetic retinopathy. 
In our opinion, histopathological studies utilizing well-known pericyte markers 3G5, NG-2 and PDGFR- $\beta$ may be more useful to determine the role of pericyte in COVID-19 pathobiology.

\section{Funding}

Support was provided solely by institutional and/or departmental sources.

\section{Compliance with ethical standards}

\section{Conflicts of interest}

The authors declare no competing interests.

\section{Research involving Human Participants and/or Animals}

This article does not contain any studies with human participants or animals performed by any of the authors.

Informed consent

For this type of study formal consent is not required.

\section{Publisher's Note}

Springer Nature remains neutral with regard to jurisdictional claims in published maps and institutional affiliations.
Accepted: 31 July 2020

Published online: 8 August 2020

\section{References}

1. Cardot-Leccia N, Hubiche T, Dellamonica J et al (2020) Pericyte alteration sheds light on micro-vasculopathy in COVID-19 infection. Intensive Care Med. https://doi.org/10.1007/s00134-020-06147-7

2. Bordenave J, Tu L, Berrebeh N et al (2020) Lineage tracing reveals the dynamic contribution of pericytes to the blood vessel remodeling in pulmonary hypertension. Arterioscler Thromb Vasc Biol 40:766-782

3. De Smedt A, Vanderlinden E, Demanet C, De Waele M, Goossens A, Noppen M (2004) Characterisation of pleural inflammation occurring after primary spontaneous pneumothorax. Eur Respir J 23:896-900

4. He L, Mäe MA, Sun Y et al (2020) Pericyte-specific vascular expression of SARS-CoV-2 receptor ACE2-implications for microvascular inflammation and hypercoagulopathy in COVID-19 patients. bioRxiv. https://doi. org/10.1101/2020.05.11.088500

5. Jain A, Doyle DJ (2020) Stages or phenotypes? A critical look at COVID-19 pathophysiology. Intensive Care Med 46:1494-1495 